\title{
High-precision two-phase laser rangefinder PFSD-1,2
}

\author{
Yegisabeth Hayrapetyan", Hovseph Petrosyan, Hovnan Hunanyan and Artyom Tsaturyan \\ National University of Architecture and Construction of Armenia, 105 Teryan st., Yerevan, 0009, \\ Armenia
}

\begin{abstract}
Performing high-precision linear measurements is one of the main tasks of modern engineering geodesy. Consequently, the development and creation of high-precision laser rangefinders and refractometers with a relative measurement error of not more than $3.10^{-7}$, becomes an urgent scientific and technical problem.

Wide theoretical and experimental studies in the problem laboratory of geodetic measurements of NUACA have accumulated a sufficient amount of experimental results for the construction of modern high-precision light meter with the determination of the residual part of the phase cycle with an error of 0.03-0.05 mm.

The article discusses issues related to improving the accuracy of linear measurements developed in the NUACA of high-precision light rangefinder. A two-phase modulation measurement method is proposed, when signals shifted by $180^{\circ}$ are formed optically using a phase plate at $\lambda / 2$. This modulation method of linear measurements provided the phase error of linear measurements $\mathrm{m}_{\varphi}=0.03-0.05 \mathrm{~mm}$.

The article also discusses the issue of reducing the modulation power. For this purpose, it is proposed to install a high-quality buffer Q-resonator between the high-quality light modem and the low-quality microwave oscillator.
\end{abstract}

\section{Introduction}

At the present stage more and more increased requirements for precision geodetic work for such special objects as Comparators, the baseline on the faults of tectonic plates, large space antennas, charged particle accelerators, etc. Performing high-precision linear measurements is one of the main tasks of modern engineering geodesy. Consequently, the development and creation of high-precision laser rangefinders and refractometers with a relative measurement error of not more than $3.10^{-7}$, becomes an urgent scientific and technical problem.

In foreign high-precision light rangefinders on the compensation method of the light electro-optical modulation, increase of the measurement precision is implemented by rehandling of the minimum demodulated light by small deviation of the modulation frequency taking place in Mekometer ME-3000 and Geomancer GR-204 [1] on the

\footnotetext{
"Corresponding author: helizabet@yandex.ru
} 
modulation frequency $500 \mathrm{MHz}$ greatly reduced the error of determining the residual part of the phase cycle equal to $\mathrm{m}_{\varphi}=0,25-0,35 \mathrm{~mm}$.

The use of the specified range-finders on various linear measurements showed the feasibility of introducing them into the work of building national geodetic networks " 0 " classis for performing measurements on the base lines with an error $\mathrm{m}_{\mathrm{s}}= \pm\left(0.1+2 \cdot 10^{-7} \mathrm{~S}\right)$ $\mathrm{mm}[2]$.

Recently, when studying the movements of the earth's crust, precision phase rangefinders, such as the Mecometer ME-5000 and ME-3000, have been successfully used, the accuracy of which is in the range of $0.3-0.5 \mathrm{~mm}$. For example, in the western part of Germany, in the seismically active zone of Zollernalb, employees of the University of Karlsruhe conducted geodesic monitoring to study the deformations using the specified rangefinders [3]. In the Russian Federation, the ME-5000 optical rangefinder [4] was also used to study the deformation on the Sami Fault (Khibiny).

These studies indicate the possibility of using optical high-precision rangefinders in geodetic monitoring in seismically active zones for estimating the deformations of the earth's crust. These measurements also indicate the need to improve the accuracy of linear measurements.

Initially, the results of control measurements on short lines of the interference comparator of the city of Sydney [1] by four samples of the ME-3000 light rangefinders and the Geomensor of the GR-204 were used. The results of repeated measurements have shown that the measurement errors of these rangefinders are larger than indicated in the operating instructions.

Therefore, it is necessary to build a " 0 " classis light rangefinder [5] that does not contain the main errors of the light-rangefinders [1], thereby initiating the development of new high-precision light-rangefinders.

The construction of the DVSD-1200M [6] and SD-1200 range finders at a modulation frequency of $1200 \mathrm{MHz}$ without transceiver optics and other optical elements in the path of modulated light showed results $\mathrm{m}_{\varphi}=0.05 \mathrm{~mm}$ [4], $\mathrm{m}_{\varphi}=0.03 \mathrm{~mm}$ [7].

Currently, in order to improve the accuracy of linear measurements, these rangefinders are being upgraded with the transition to a two-phase measurement method, the expected accuracy of which should be in the range of $\mathrm{m}_{\varphi}=0.01-0.02 \mathrm{~mm}$.

Analysis of the measurement results revealed that it is impossible to obtain highaccuracy measurements with $\mathrm{m}_{\varphi} \approx 0.01 \mathrm{~mm}$ using the compensation method, since the modem's operating point lies at the beginning of the Light Modulation Characteristic (LMC) [8], which means low reception sensitivity, high modulation power that violates the modem's crystal structure, and large non-linear distortions. All this can be eliminated by switching to a two-phase method of modulating light, which at low frequencies is realized by reversing the phase of the modulation frequency by $180^{\circ}$ electrically. In the ultra-high frequency (UHF) range, the phase-shifted $180^{\circ}$ paired signals are formed in the optical channel [9] based on the natural property of the KDP crystal (potassium dihydrogen phosphate).

\section{Methods}

All this can be eliminated by a transition to two-phase modulation method of light. At low frequencies, the modulation phase shift on $180^{\circ}$ is realized electrically. On the UHF range phase shifted on $180^{\circ}$ signals are formed in the optical channel [9] based on the natural property of the KDP crystal (potassium dihydrophosphate). 
A known property of crystal KDP - shift of the modulation phase of the light $180^{\circ}$ by changing the position of polarization of the light $90^{\circ}$, allows to periodically change the phase of light modulation by use of phase plate on $\lambda / 2$ at the output of the light modem.

The solution of the matrix equation of the light modulation-demodulation at linear polarization of light and the installation of the phase plate at $\lambda / 2$ for the relative intensity of light at the output of the analyzer leads to the expression of the following form

$$
\begin{aligned}
& I / I_{0}=\frac{1}{2}\left[1-\frac{1}{2}\left(\cos \Gamma_{1}+\cos \Gamma_{1} \cos \Gamma_{2}\right)+\frac{1}{4} \cos \Gamma_{1}\left(1-\cos \Gamma_{2}\right) \cdot \cos 8 \theta-\right. \\
& \left.-\frac{1}{2} \sin \Gamma_{1} \sin \Gamma_{2} \cos 4 \theta\right]
\end{aligned}
$$

where $\Gamma_{1}$ is the phase shift when the light is modulated, $\Gamma_{2}$ is the phase shift when light is demodulated, $\theta$ is the angle of rotation of the phase plate by $\lambda / 2$.

For expression (1), the main options are $\theta=0$ and $\theta=45^{\circ}$.

1. When $\theta=0$ or $\theta=90^{\circ}$, we have

$$
I / I_{0}=-\sin ^{2} \frac{\Gamma_{2}-\Gamma_{1}}{2}=\frac{1-\cos \left(\Gamma_{2}-\Gamma_{1}\right)}{2}
$$

This is the basic expression of the compensation measurement method. In this case, one of the axes $n_{0}$ and $n_{e}$ of the phase plate coincides with the direction of light polarization and there is no rotation of the light polarization.

2. $\theta=45^{\circ}$

$$
I / I_{0}=\sin ^{2} \frac{\Gamma_{2}+\Gamma_{1}}{2}=\frac{1-\cos \left(\Gamma_{2}+\Gamma_{1}\right)}{2}
$$

Now the position of the minimum of the ratio $I / I_{0}$ is shifted by the value $\lambda_{\mathrm{m}} / 4$, i.e. the light polarization with the axes $n_{0}$ and $n_{e}$ of phase plate makes an angle of $45^{\circ}$ and is split into two components along the $n_{0}$ and $n_{e}$ axes, the phase shift between which is $180^{\circ}$ which results in a $90^{\circ}$ rotation of the polarization. This is equivalent to turning the KDP crystal around the optical axis $\mathrm{Z}$ by $90^{\circ}$ while maintaining the same polarization of light. When periodically passing the light of $0^{\circ}$ and $90^{\circ}$ polarizations through the light demodulation crystal, we alternately receive signals (2) and (3), the equality of which comes under the condition $\cos 2 \pi S / \lambda_{\mathrm{m}}=\sin 2 \pi S / \lambda_{\mathrm{m}}$ that occurs when $S=\lambda_{\mathrm{m}} / 8$, i.e. the formula for measuring the two-phase method is periodic $\lambda_{\mathrm{m}} / 4$

$$
S=N \lambda_{\mathrm{m}} / 4+\lambda_{\mathrm{m}} / 8
$$

The power mode $\mathrm{U} / \mathrm{U}_{\pi}$ of the light modem providing the condition $\cos 2 \pi S / \lambda_{\mathrm{m}}=\sin 2 \pi S / \lambda_{\mathrm{m}}$ determines the power mode of $\mathrm{U} / \mathrm{U}_{\pi}$ of the light modem on the two-phase method.

$$
2 \pi U / U_{\pi} \cos 2 \pi S / \lambda_{\mathrm{m}}=2,4 \text { at } \mathrm{S}=\lambda_{\mathrm{m}} / 8 \text { or } 2 \pi U / U_{\pi} \cdot 0.7=2.4 ; U / U_{\pi}=0.54
$$

At $U / U_{\pi}=0.54$, the modulation power at a frequency of $1200 \mathrm{MHz}$ remains high $\mathrm{P}_{\mathrm{m}}=$ $130 \mathrm{~W}$ and at such modulation powers in the pulses with a duration of about $40 \mu \mathrm{s}$ crystals introduce phase distortions of $0.1-0.15 \mathrm{~mm}$ from heating. Therefore, it is important to reduce the UHF power in the two-phase light rangefinders.

The position of the ODL (Optical Delay Line) is fixed at $\mathrm{S}=\lambda_{\mathrm{m}} / 8$, when the intensities of the two-phase light components become equal.

In order to improve the accuracy of measurements, an increase in the modulation frequency in the optical rangefinder is impractical, since this increases the modulation power, so the pulsed mode is used. Despite this, at pulse durations of 30-40 $\mu$ s, crystal heating is observed, leading at the front of the receiving light to phase variations, which is 
an additional source of measurement error. The use of moving of the light modem, as an ODL, is not the best solution, since affects the value of the stadia constant. The installation of transceiver optics, even of small diameter, is necessarily; this was revealed in $[10,11]$ with a decrease $\mathrm{m}_{\varphi}$ in 1.5-2 times.

Thus, the implementation of the two-phase method by rotating the phase plate by $\lambda / 2$, obtaining $\mathrm{m}_{\varphi}=0.03 \mathrm{~mm}$ without reducing the modulation power does not lead to the implementation of linear measurements with an accuracy of $m_{s}=\left(0.1+2 \cdot 10^{-7} \mathrm{~S}\right) \mathrm{mm}$.

Given all this, to build a new high-precision light rangefinder, it is necessary to perform the following basic changes:

1. The transition from the compensation method of electro-optical modulation to the two-phase method by turning the phase plate on $\lambda / 2$, installed in front of the light demodulation channel.

2. Fixed installation of a modem of light, construction of ODL on a corner prism and reduction of the length of the ODL motion twice using the two-phase method.

3. Separation of the light modulation and demodulation channels within 14-16 mm by application of a biaxial light modem [12] on crystals with a cross section of $6 \times 6 \mathrm{~mm}^{2}$ and a length reduced from $35 \mathrm{~mm}$ to $20 \mathrm{~mm}$.

4. To exclude the direct connection of the low-quality microwave generator $\mathrm{Qg}=300$ with a high quality light modem, $\mathrm{Q}_{\mathrm{m}}=1000$. This leads to the fact that at a frequency of $1200 \mathrm{MHz}$, in order to obtain $100 \%$ light modulation efficiency; the necessary modulation power is about $180 \mathrm{~W}$, which is unacceptable in a portable device.

5. A microwave generator scale frequency of $1200 \mathrm{MHz}$ should be constructed based on a quartz oscillator at $600 \mathrm{MHz}$ by multiplying the frequency up to $1200 \mathrm{MHz}$ and forming a modulation power of no more than $40-50 \mathrm{~W}$.

6. It is necessary to use a new solution to reduce the modulation power by installing an additional high-quality resonator with $\mathrm{Q}=2000$ between the microwave generator and the light modem.

7. Installation of receiving-transmitting optics, increasing the intensity of the receiving light, which not only leads to a decrease in modulation power, but also averages the phase dispersion at the front of the receiving light.

8. Transition from the coaxial resonator of the light modem to the biaxial one, applying the property of the coaxial resonator when the internal conductor is displaced from the resonator axis and the second conductor is introduced symmetrically.

In the capacitance gap of the anaxial cavity, the maximum electric field $E_{01}$ shifted with the internal conductor, and the resonator resonance frequency is reduced.

The placement of the second conductor in the resonator further reduces the resonance frequency of the already biaxial resonator excited on the $\mathrm{E}_{02}$ wave, the maximum of which is evenly distributed around each conductor of the resonator. Increasing the quality factor of the biaxial modem and reducing the cross section of the crystals preserves the efficiency of light modulation in the biaxial modem at the $\mathrm{E}_{01}$ energy level of the coaxial resonator.

The functional diagram of the new two-phase rangefinder PFSD-1.2 is shown in Figure 1 and works as follows.

The emission of a laser type LGN-207A 1 polarized linearly, by mirrors 2 of the outer coating is sent into a KDP 3 crystal mounted coaxially at the end with an internal conductor displaced from the axis of the resonator 4 by $8 \mathrm{~mm}$. Phase-modulated light by transmitting optics 5 with a lens diameter of about $20 \mathrm{~mm}$ is directed to the reflector mounted at the end of the measured line.

The Central part of the reflected light flux with a diameter of about $30 \mathrm{~mm}$ by the receiving optics 6 is compacted into a parallel light flux with a diameter of $2.5 \mathrm{~mm}$ and through the free volume of the resonator 4 is directed to the OLZ, built on a prism 7 of the angular type, the movement of which within $75 \mathrm{~mm}$ is provided by the guides 9 with a 
carriage 8 on which the rack 11 connected to the worm gear 12 is installed. One revolution of the worm axis moves the ODL in $2 \mathrm{~mm}$. The position of the ODL is fixed on the digital display 14 .

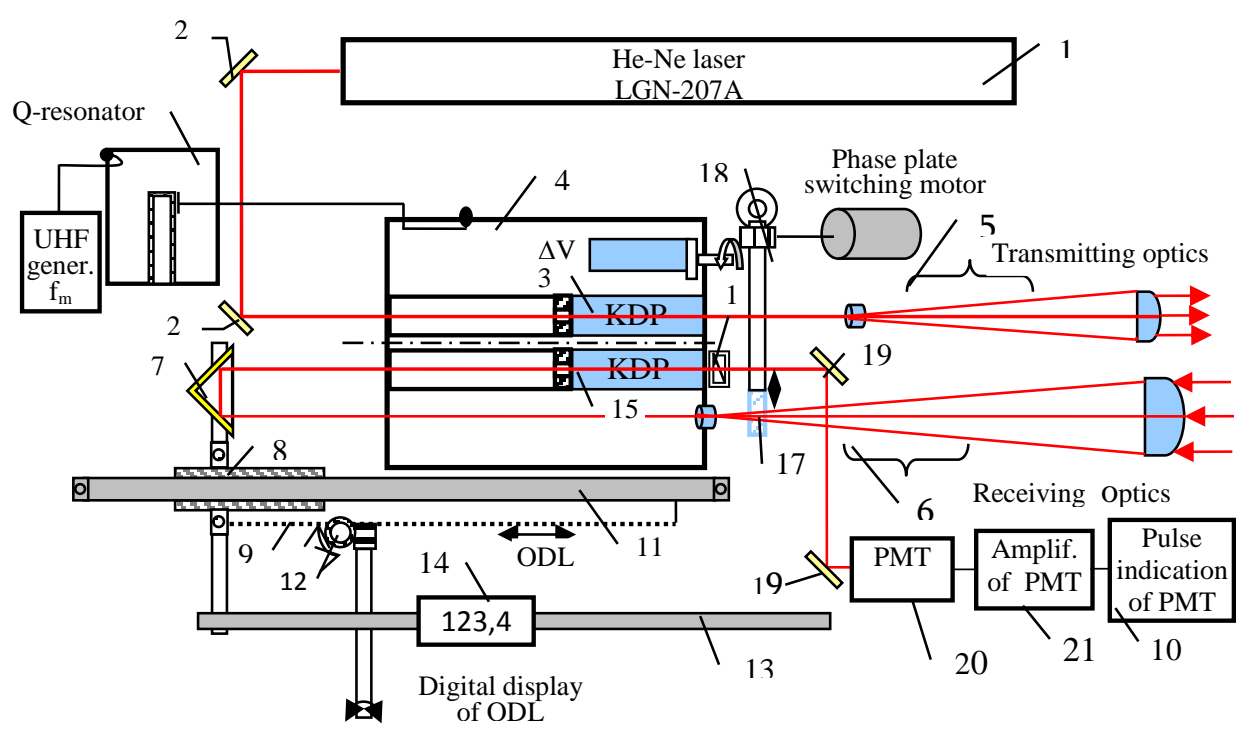

Fig.1 Functional scheme of the two- phase laser rangefinder PFSD-1.2.

The movement of the prism $7 \mathrm{ODL}$ is determined with an error of $0.01 \mathrm{~mm}$. The reflected light flux from the prism 7 is offset from the receiving flux by $8-10 \mathrm{~mm}$ and directed into the crystal 15 of the demodulator mounted on the second conductor of the resonator symmetrically to the first. At the output of the crystal 15, the analyzer 16 and the phase plate at $\lambda / 217$ are installed. Switching of the phase plate 17 by the mechanism 18 from the demodulated light after the analyzer to the modulated at the output of the receiving lens leads to the transition to a two-phase modulation method. The points of light minima arriving at the $n \lambda_{m} / 4$ position of the ODL prism7, by introducing the phase plate at $\lambda / 217$ after the receiving lens, the modulation phases shift by $180^{\circ}$ and the minima of the demodulated light on the photomultiplier tube (PMT) 20, come to the maxima of the modulated light. By moving the prism on a length $\lambda_{\mathrm{m}} / 4$, the maxima of light observed on the screen 10 become minima, i.e., when moving the prism 7 equal to $\lambda_{\mathrm{m}} / 8$, the intensities of the incoming phase-shifted light fluxes become equal. At this point, the modulation voltage becomes " 0 ", those demodulation does not occur. The shift of the ODL from this position linearly changes the amplitudes of the receiving signals, equal to half the total intensity of the light.

Thus, there is a decrease in the travel path of ODL by 2 times, an increase in the intensity of the receiving light and a linear dependence on the applied voltage on the demodulation crystal sharply increases the sensitivity of determining the position of fixed signals. The two-phase method within small limits reduces the power of microwave modulation and, most importantly, reduces the error in determining the phase $m_{\varphi}$ within 10 times. On a line longer than $100 \mathrm{~m}$, a mirror-lens reflector of the focusing type can become the main one for the new light-rangefinder, and a flat mirror with a diameter of 100-120 $\mathrm{mm}$ with an external coating can be used up to $100 \mathrm{~m}$ [13].

Some reduction of modulation power is not enough to reduce the phase dispersion at the front of the modulated light to $\mathrm{m}_{\varphi}=0.01 \mathrm{~mm}$ in the two-phase mode. 


\section{Discussion}

The first ways to reduce the modulation power are to reduce the modulation frequency and increase the light intensity. On Fig.2 the light demodulation curves at $600 \mathrm{MHz}$ and at 1200 $\mathrm{MHz}$ are compared with a 2 -fold decrease in modulation power, i.e. at the same modulation power, and it is seen that the sensitivity at $1200 \mathrm{MHz}$ is greater than $600 \mathrm{MHz}$, i.e., at the same ODL displacements, the signal reduction is 1.25 times less by $600 \mathrm{MHz}$. This means that at high frequencies it is expediently to apply other methods for significantly reduce the modulation power.

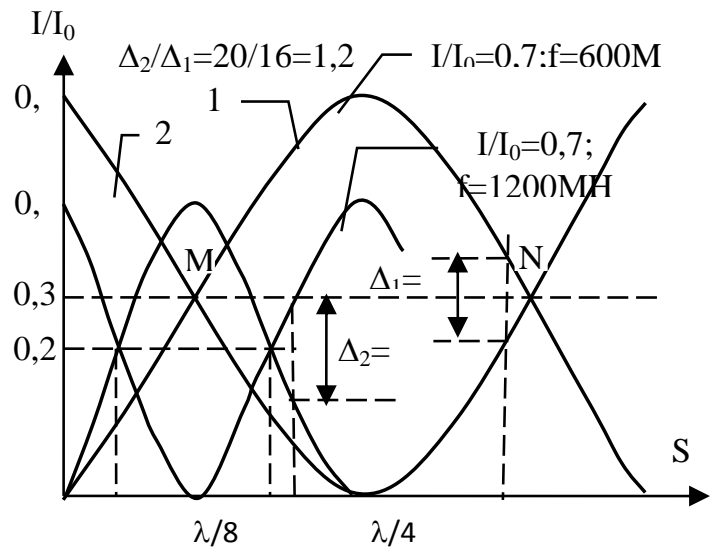

Fig. 2. With the same modulation power $\mathrm{P}=60 \mathrm{~W}$ at a frequency of $1200 \mathrm{MHz}$, the sensitivity is 1.25 times more

From the expression of the required modulation power

$$
P_{m}=U^{2} \omega_{m} c_{r} / 2 Q_{L},
$$

it follows that in order to reduce $\mathrm{P}_{\mathrm{r}}$, it is necessary to decrease the capacity of the load $\mathrm{C}_{\mathrm{r}}$ and to increase the quality factor $\mathrm{Q}_{\mathrm{L}}$ of the load connected to the generator.

A coaxial volume resonator with a small gap capacity of about $0.2-0.25 \mathrm{pF}$ at 1200 $\mathrm{MHz}$ frequency ensures the quality factor of order $\mathrm{Q}_{\mathrm{L}} \geq 2000$. Installing such a resonator between a light modem and a microwave generator will get a cascade inclusion of resonators applied in microwave circuits of various purposes. The basic concepts of a resonator with one coupling element can be transferred with a small transformation to a resonator with two coupling elements connected in a pass-through scheme. In such a system, the phenomenon of resonance is characterized by a high level of energy accumulated in the resonator. The sharpness of the resonance caused by the loaded quality factor $\mathrm{Q}_{\mathrm{L}}$, which is expressed by the quality factor $\mathrm{Q}_{0}$, which determines the losses in the resonator, and the external quality factor $\mathrm{Q}_{\mathrm{ex}}$, which characterizes the connection of the resonator with other circuits.

External Q-factor $\mathrm{Q}_{\mathrm{ext}}$ is an artificial concept and has no relation to practice. External quality factor depends on $\mathrm{n}^{2}$ coupling value $[14,15]$

$$
Q_{\text {ext. }}=\frac{Z_{0}}{n^{2}} \cdot \sqrt{\frac{c_{e q .}}{L_{e q .}}},
$$

where $\mathrm{Z}_{0}$ is the input resistance, $\mathrm{C}_{\mathrm{eq}}$., $\mathrm{L}_{\mathrm{eq}}$ is equivalent capacitance and inductance.

Loaded $\mathrm{Q}_{\mathrm{L}} \mathrm{q}$-factor is also, by definition, to some extent, an artificial concept. The physical meaning of the definition lies first in the fact that the $\mathrm{Q}_{\mathrm{L}}$ value determines the time constant of the all system and the bandwidth of the system corresponding to the $\mathrm{Q}_{\mathrm{L}}$ value by the frequencies of the half power of the resonator. 


$$
Q_{L}=\omega_{0} / \Delta \omega_{L},
$$

where $\omega_{0}$ is the main resonant frequency, $\Delta \omega_{\mathrm{L}}=\omega_{2 \mathrm{~L}}-\omega_{1 \mathrm{~L}}$ are frequencies of half power.

The loaded $\mathrm{Q}$-factor $\mathrm{Q}_{\mathrm{L}}$ does not depend on the connection and depends only on the loss of $\alpha_{T}$;

$$
Q_{0}=\bar{c} r \omega_{0} \text { or } Q_{0}=\frac{\omega_{0}}{\omega_{2}-\omega_{1}}=\frac{\omega_{0}}{\Delta \omega_{0}} .
$$

It is obvious that significant differences in the characteristics of the transmission for the two-stage resonators, and the United separately one of the resonator should not be, only at cascade connection, the transmission characteristic is sharper, since the $\mathrm{Q}_{\mathrm{L}}$ is twice as large.

If both resonators are at comparable $\mathrm{Q}_{0}$, then the total unloaded q-factor of $\mathrm{Q}_{\text {tuL }}$. will be more than for each individual resonator. For two identical resonators $\mathrm{Q}_{\text {ofull }}=\mathrm{Q}_{0}$; $\mathrm{Q}_{\mathrm{tuL}}=$ $2 \mathrm{Q}_{\mathrm{ext}}$, therefore

$$
Q_{L_{\text {full }}}=\frac{Q_{\text {ext }} \cdot Q_{L}}{Q_{\text {ext }}-Q_{L}} .
$$

In the usual expression, instead of the "-" sign, there is a " + " sign in front of $Q_{L}$, therefore the cascade half-wave inclusion of two resonators increases the stored energy and always leads to an increase in $\mathrm{Q}_{\mathrm{ext}}$. This shows the need for a Q-resonator in the circuit in Fig.1, and this scheme of high precision light rangefinder will provide the phase error $\mathrm{m}_{\varphi}=0.01 \mathrm{~mm}$.

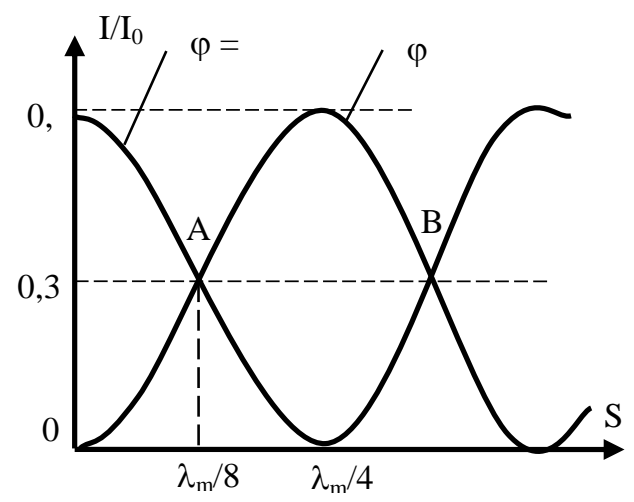

Fig.3 Dependence of two-phase signal on distance

At the output of the crystal 15 demodulator, the output level of the light is determined by the amplitude of the light in the crystal 3 and the losses on the ODL prism. Figure 3 shows the dependence of the two-phase signal on the distance, and Figure 4 - the type of receiving two-phase pulses on the oscilloscope screen. Indication occurs when the pulse amplitudes are equal. At the output of the photomultiplier tube (PMT) 20, modulation impulses depend on the degree of demodulation shown in Fig.3. In the ODL position, equal to $\lambda_{\mathrm{m}} / 8$, the amplitudes of two-phase signals are equal and their equality can be seen on screen 10 connected to the output of the PMT amplifier 21. The use of receiving and transmitting optical system and transfer the position of the equality of the amplitudes of the two-phase of the signals at the middle half of the light intensity (points $M, N$ in Fig.2), form the large light intensity on the PMT in a small diameter $\mathrm{D}=2.5 \mathrm{~mm}$. This allows the use of small diameter PMT and expand the beam of light on the photocathode to a diameter of 8 $10 \mathrm{~mm}$, using the all area of the PMT photocathode. 


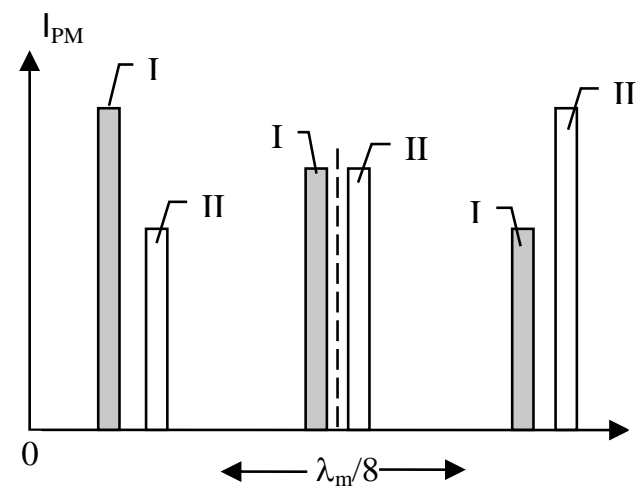

Fig. 4. The amplitude of the two-phase pulses in different positions of ODL

The pulses periodicity the of equal amplitudes at the points $\mathrm{M}, \mathrm{N}$ of the curve crossing in Fig. 2 determines the formula for measuring the distance on the two-phase method

$$
S=N \frac{\lambda}{4}+\frac{\lambda}{8}+\Delta \ell-K
$$

where $\mathrm{N}$ is the integral number of $\lambda_{\mathrm{m}} / 4$ at the measured distance, $\Delta \ell$ is the value of the residual part of the phase cycle; $\mathrm{K}$ is the constant correction of the laser rangefinder.

During the measurement, the temperature and pressure are determined along the line to be measured by conventional methods.

\section{Results}

The results of the study of the layout show the possibility of developing a light meter on a two-phase method with an error $\mathrm{m}_{\varphi}=0.02-0.03 \mathrm{~mm}$. Such a device can also serve as an intermediate when transmitting a unit of length from the standard to the measuring instruments and with some modernization the device can become a rangefinder " 0 "class.

\section{Conclusion}

For the rangefinder, built according to the scheme in Fig.1 applicable method of selfcertification of device by 4-5 segments of the same line.

In conclusion, we note that the light rangefinder, built according to the scheme in Fig. 1, is much more economical and reliable than interferometers of various modifications and can be used along with interferometers at various high-precision works, regardless of weather conditions.

The work was carried out within the framework of the state budget financing of the problem laboratory of geodetic measurements of NUACA on the theme "Scientific research and monitoring of the earth's crust displacement in seismically active zones using modern technologies and highprecision devices".

\section{References}

1. J.M. Ruger and P.E. Ciddor. The Australian surveyer, Iuna, vol.33, №6, p.p. 480492(1987),

2. G.A. Ustavich, Geodesy, (1999), p. 6-15. 
3. K. Zippelt, Proceedings 11th Int. FIG Symposium on Deformation Measurements, Santorini (Thera) Island, Greece, May 25-28 2003, Publication No. 2, Geodesy and Geodetic Applications Lab. Dept. of Civil Engineering, Patras University (2003), p.5158.

4. A.A. Kozyrev, E.V. Kasparyan, D.B. Zhirov, Yu.G. Smagina, Bulletin of MGTU, volume 12, N4, (2009), p.702-707.

5. A.G. Beglaryan, K.S. Gyunashyan, Ye.H. Hayrapetyan, Khachatryan K.Kh., Geodesy and aerial photography, №2, (2005).

6. R. A. Movsesyan, K. S. Gyunashyan, Kh. S. Mehrabian, V. Y. Weinberg, Geodesy and aerial photography, №1, (1981).

7. A.G. Beglaryan, K.S. Gyunashyan, Ye.H. Hayrapetyan Proceedings of 3-rd int. confer. On contemporary problems in archit. And construction. Beijing, China, Nov. 20-24, (2011), p.9-14

8. E. R. Mustel, V. N. Parygin, Methods of modulation and scanning of light. M. Nedra, (1970) $296 \mathrm{p}$.

9. K.S.Gyunashyan, Ye.H. Hayrapetyan. Patent 1598613 (USSR). In B.I.,(1990), №37

10. V.Ya. Weinberg, F.V. Shirov, Geodesy and cartography (1983), №4, p.17-22

11. H. Potthoff, Vermessungstechnik 28, Heft 10, (1980), s.325-328

12. K. Khachatryan, V.Harutyunyan, H. Nersisyan, NUACA collection of scientific works, (2011), vol. 2, p.115- 121.

13. K.S.Gyunashyan, Ye.H. Hayrapetyan, Markaryan V.A. News Of The Academy Of Sciences. USSR ser. Technical science, XIV №4, (1991), p.193-200.

14. G. Megla. Decimeter wave technique. M., Sovetskoe radio, (1958), 462p.

15. A.I. Astaykin, K.V. Trotsyuk, S.P. Ionova, V.B. Profe (2008) Teoreiya i tekhnika SVCH. Sarov, 466p. 